\title{
Pluripotential theory on the support of closed positive currents and applications to dynamics in $\mathbb{C}^{n}$
}

\author{
Frédéric Protin ${ }^{1}$
}

Received: 8 May 2018 / Accepted: 29 March 2019 / Published online: 5 April 2019

(c) Fondazione Annali di Matematica Pura ed Applicata and Springer-Verlag GmbH Germany, part of Springer Nature 2019

\begin{abstract}
We extend certain classical theorems in pluripotential theory to a class of functions defined on the support of a $(1,1)$-closed positive current $T$, analogous to plurisubharmonic functions, called $T$-plurisubharmonic functions. These functions are defined as limits, on the support of $T$, of sequences of plurisubharmonic functions decreasing on this support. We study these functions by means of a class of measures, so-called pluri-Jensen measures, which prove to be numerous on the support of $(1,1)$-closed positive currents. For any fat compact set, we obtain an expression of its relative Green's function in terms of pluri-Jensen measures and deduce a characterization of the polynomially convex fat compact sets and of pluripolar sets. These tools are then used to study dynamics of a class of automorphisms of $\mathbb{C}^{n}$ which naturally generalize Hénon's automorphisms. We obtain an equidistribution result for the convergence of pull-back of certain measures toward an ergodic invariant measure with compact support.
\end{abstract}

Keywords Pluricomplex Green's function - Closed positive current · Pluripolar set . Polynomial convexity · Complex dynamics · Jensen measure

Mathematics Subject Classification 32U15 $\cdot 32 \mathrm{E} 20 \cdot 32 \mathrm{U} 35 \cdot 32 \mathrm{U} 40 \cdot 32 \mathrm{U} 05 \cdot 32 \mathrm{H} 50$

\section{Introduction}

The main goal of this article is to develop a pluripotential theory for $T$-plurisubharmonic functions (abbreviated $T$-psh), i.e., functions that are limits, on the support of a $(1,1)$-closed positive current $T$, of sequences of plurisubharmonic functions decreasing on this support. The $T$-psh functions were introduced in [3] (see also [11]), and a more general class of functions, called plurisubharmonic in the direction of the current $T$, is considered in [3] and [27] from a different point of view. $T$-psh functions naturally arise in complex dynamics, as in [22], or in [7] and [5] where they appear with a different name. The second goal of this article is to apply these results to the study of the dynamics of a certain class of automorphisms of $\mathbb{C}^{n}$

Frédéric Protin

fredprotin@yahoo.fr

1 INSA de Toulouse, Institut de Mathématiques de Toulouse, 135 avenue de Rangueil, 31400 Toulouse, France 
which generalizes Hénon's automorphisms of $\mathbb{C}^{2}$, that we call Hénon-like automorphisms. The article is structured as follows.

In Sect. 2, we develop a pluripotential theory for continuous $T$-psh functions. We proceed as follows. In Sect. 2.1, we give a more intrinsic characterization of such functions. More precisely, we prove that a continuous function $u: \operatorname{supp}(T) \rightarrow \mathbb{R}$ is $T$-psh if and only if it satisfies $u(x) \leq \int_{\operatorname{supp}(T)} u \mathrm{~d} \mu_{x}$ for every $x$ in the support $\operatorname{supp}(T)$ of $T$ and every pluriJensen measure $\mu_{x}$ relative to $x$ and with support in $\operatorname{supp}(T)$. We have equality in the previous relation when we take the infimum over the set of all such pluri-Jensen measures. We also prove that there are sufficiently many such measures in order to construct a potential theory, analogous to the one for plurisubharmonic functions. We deduce some results, as for example a maximum principle for $T$-psh functions, and the fact that a support of a closed positive $(1,1)$-current is nowhere pluri-thin.

In Sect. 2.2, for an open connected bounded hyperconvex set $U \subset \mathbb{C}^{n}$, we express the value at any $x \in U$ of the Green's function relative to $U$ of a fat compact set $A \subset U$ as the infimum of $-\mu_{x}(\AA)$ over all pluri-Jensen measures $\mu_{x}$ relative to $x$ with support in $U$. We deduce a characterization of polynomially convex fat compact sets and of pluripolar sets in terms of pluri-Jensen measures. We further prove that the poles of $T$-psh functions form a pluripolar set.

In Sect. 2.3, we pursue the analogy between $T$-psh functions and plurisubharmonic functions. In particular, we obtain a Hartogs-like theorem, an extension property across certain small sets, and a Chern-Levine-Nirenberg inequality. We also prove that, under minor conditions, the limit superior of a locally uniformly bounded from above sequence of $T$-psh functions is a $T$-psh function, except on a pluripolar set.

In Sect. 3, we apply the previous results to the study of the dynamics of Hénon-like automorphisms. In Sect. 3.2, we give an equidistribution lemma for the pull-backs of certain closed currents by a Hénon-like automorphism, that we further exploit to obtain information about its Julia set.

In Sect. 3.3, we give an equidistribution result for the pull-backs of certain measures by a Hénon-like automorphism. More precisely, we prove that the pull-backs of a probability measure defined on the support of certain currents and which does not charge pluripolar sets, converge in a Cesàro sense toward an unique canonical invariant measure. This measure is ergodic, with compact support.

Notations. We will denote $d:=\partial+\bar{\partial}$ the usual exterior derivative and $d^{c}:=\frac{1}{2 i \pi}(\partial-\bar{\partial})$. Thus, $d d^{c}=\frac{i}{\pi} \partial \bar{\partial}$. These operators will be understood in the sense of currents.

A continuous function $u$ from an open set of $\mathbb{C}^{n}$ into $\mathbb{R}$ is pluriharmonic (harmonic if $n=1$ ) if and only if $d d^{c} u=0$. We denote by $\|S\|:=S \wedge \omega^{n-1}$ the trace measure of a closed positive $(1,1)$-current $S$ on $\mathbb{C}^{n}$, where $\omega:=\frac{1}{2} d d^{c} \log \left(1+\|z\|^{2}\right)$ is the Fubini-Study form. Then, the mass of the current $S$ is $\|S\|\left(\mathbb{C}^{n}\right)$.

\section{Study of $T$-plurisubharmonic functions}

\subsection{Fundamental results on $T$-psh functions}

In the sequel, we denote by $\hat{A}$ the polynomially convex envelope of a compact $A$. Following [11], we define the $T$-psh functions as follows:

Definition 1 Given a closed positive $(1,1)$-current $T$ defined in an open set $V \subset \mathbb{C}^{n}$ with support $\mathcal{S} \subset V$, a function $u: \mathcal{S} \rightarrow \mathbb{R} \cup\{-\infty\}$ is called $T$-plurisubharmonic (abbreviated 
$T$-psh) if it is locally $\|T\|$-integrable and if there exists an open set $U \subset V$ containing $\mathcal{S}$, and a sequence of plurisubharmonic functions $u_{m}: U \rightarrow \mathbb{R}$ decreasing on $\mathcal{S}$, such that $\left(\left.u_{m}\right|_{\mathcal{S}}\right)_{m}$ converges simply toward $u$ on $\mathcal{S} \cap U$. Up to shrinking $U$, we may suppose that the functions $u_{m}$ are continuous.

More generally, if we replace $\mathcal{S}$ by a compact $K \subset \mathbb{C}^{n}$ in the previous definition, the function $u: K \rightarrow \mathbb{R} \cup\{-\infty\}$ will be said $K$-plurisubharmonic.

The elements of a family $\left(v_{m}\right)_{m \in \mathbb{N}}$ of $T$-psh functions will be said co-T-psh if for every $m \in \mathbb{N}$, there exists an open set $U \subset V$ containing $\mathcal{S}$, and a sequence of plurisubharmonic functions $\left(v_{m, i}\right)_{i}: U \rightarrow \mathbb{R}$ decreasing on $\mathcal{S} \cap U$ toward $v_{m}$.

The current $T \wedge d d^{c} u:=d d^{c}(u T)$ is then well defined, positive and closed (see Lemma $4-13$ in [11]).

The main purpose of this section is to present a more intrinsic characterization of continuous $T$-psh functions. First let us recall some definitions and notations.

A pluri-Jensen measure $\mu_{x}$ relative to $x \in \mathbb{C}^{n}$ is a regular probability measure with compact support such that, for any plurisubharmonic function $v$ defined on an open set $U$ containing $x$, we have

$$
v(x) \leq \int_{U} v \mathrm{~d} \mu_{x} .
$$

If $v$ is only assumed subharmonic, $\mu_{x}$ is a Jensen measure relative to $x$. (Therefore, it is also a pluri-Jensen measure.) The reader should pay attention to the fact that a pluri-Jensen measure in our terminology is called Jensen measure in [28,29], and [17].

Remark 1 It is sufficient to suppose that (1) holds when $v$ is a continuous plurisubharmonic function in order to ensure that $\mu_{x}$ is a pluri-Jensen measure, thanks to the monotone convergence theorem, since a plurisubharmonic function is a limit of a decreasing sequence of continuous plurisubharmonic functions in a neighborhood of the support of $\mu_{x}$. It is even sufficient to suppose that $v$ is of the form $\log |f|$ for some holomorphic function $f$, since a classical theorem of Bremermann ensures that a continuous plurisubharmonic function can be approximated locally uniformly by functions of the form $\max _{i=1, \ldots, m} c_{i} \log \left|f_{i}\right|, c_{i}>0$, where $f_{i}$ are holomorphic functions.

Let $B \subset \mathbb{C}^{n}$. We denote by $P J_{B}(x)$ the set of pluri-Jensen measures relative to $x$ with support in $B$. Note that $\delta_{x} \in P J_{B}(x)$ if $x \in B$. Moreover, for a given compact set $A \subset \mathbb{C}^{n}$, for any $x \in \hat{A}$, there exists a measure $\mu_{x} \in P J_{A}(x)$. (This follows from Theorem 1.2.9, Theorem 1.2.14 and Remark 1.2.18 in [28], or even directly from Theorem 2.2.5 in [28], see also [8].) Note also that an automorphism $Q: \mathbb{C}^{n} \rightarrow \mathbb{C}^{n}$ induces a bijection $Q_{*}$ : $P J_{\mathbb{C}^{n}}(x) \rightarrow P J_{\mathbb{C}^{n}}(Q(x))$.

The following lemma ensures the existence of sufficiently many pluri-Jensen measures on the support of a closed positive current, allowing us to establish a potential theory on it.

Lemma 1 Let $T$ be a closed positive $(1,1)$-current in $\mathbb{C}^{n}$, of support $\mathcal{S}$. Then, $\forall r>0$, $\forall x \in \mathcal{S}$, there exists a pluri-Jensen measure relative to $x$ with support in $\partial B(x, r) \cap \mathcal{S}$, where $B(x, r)$ denotes the open ball of center $x$ and radius $r$.

Proof Let $y \in \partial B(x, r) \cap \mathcal{S}$. Then, $y$ is a peak point for the uniform algebra $\mathcal{A}$ of the functions on $\bar{B}(x, r) \cap \mathcal{S}$ with complex values that are restrictions of holomorphic functions defined in a neighborhood of $\bar{B}(x, r)$ : in other words, there exists $f \in \mathcal{A}$ such that $f(y)=1$ and $|f(z)|<1$ for $z \in(B(x, r) \cap \mathcal{S}) \backslash\{y\}$. 
Indeed, assuming that $x$ is the origin, the restriction of the function $f(z)=\frac{1}{2}(1+<$ $\left.z, \frac{y}{\|y\|}>\right)$ to $\bar{B}(x, r) \cap \mathcal{S}$ fulfills these conditions. $(<\cdot, \cdot>$ denotes here the canonical hermitian scalar product on $\mathbb{C}^{n}$.)

In particular, $y$ is a strong boundary point for $\mathcal{A}$, i.e., for any neighborhood $V$ of $y$ for the topology induced on $\partial B(x, r) \cap \mathcal{S}$, there exists $f \in \mathcal{A}$ such that $\sup _{V}|f|=f(y)=1$ and $|f(z)|<1$ when $z \in(B(x, r) \cap \mathcal{S}) \backslash V$.

On the other hand, there is no strong boundary point for $\mathcal{A}$ in $B(x, r) \cap \mathcal{S}$. Indeed, suppose that there exists such a point $z \in B(x, r) \cap \mathcal{S}$. The point $z$ cannot be a peak point, since it follows from Proposition 2.5 in [6] that the algebra $\mathcal{A}$ cannot have local peak points in $B(x, r) \cap \mathcal{S}$. Thus, for any neighborhood $V$ of $y$, a function $f \in \mathcal{A}$ such that $\sup |f|=$ $f(y)=1$ and $|f(z)|<1$ for $z \in(B(x, r) \cap \mathcal{S}) \backslash\{y\}$ takes the value 1 on $V \backslash\{z\}$. By the Theorem of isolated zeros applied to the holomorphic function whose restriction is $f$, it follows that $f$ is constant. Consequently, $z$ cannot be a strong boundary point.

Therefore, the strong boundary points of $\mathcal{A}$ belong to $\partial B(x, r) \cap \mathcal{S}$. But by Corollary 7.24 in [29], the Shilov boundary $\Gamma$ for $\mathcal{A}$ is the closure of the set of strong boundary points of $\mathcal{A}$, and thus $\Gamma \subset \partial B(x, r) \cap \mathcal{S}$. Then, by Lemma 6.2 in [1] and Remark 1, there exists a pluri-Jensen measure relative to $x$ with support in $\Gamma$ (see also Remark 1.2.18 in [28]).

Remark 2 Let $T$ be a closed positive $(1,1)$-current in an open subset of $\mathbb{C}^{n}$, with support $\mathcal{S}$, and $u: \mathcal{S} \rightarrow \mathbb{R}$ a $T$-psh function. The monotone convergence theorem and Lemma 1 imply that $\forall x \in \mathcal{S}, \forall v_{x} \in P J_{\mathcal{S}}(x)$, we have $u(x) \leq \int_{\mathcal{S}} u \mathrm{~d} v_{x}$. Since $\delta_{x} \in P J_{\mathcal{S}}(x)$, we obtain

$$
u(x)=\inf _{v_{x} \in P J_{\mathcal{S}}(x)} \int_{\mathcal{S}} u \mathrm{~d} v_{x} .
$$

We deduce a maximum principle for $T$-psh functions which improves Lemma 4.3 in [22] (see also Theorem 3.2 in [27]):

Corollary 1 Let $T$ be a closed positive $(1,1)$-current in $\mathbb{C}^{n}$, of support $\mathcal{S}$, and $u: \mathcal{S} \rightarrow \mathbb{R} a$ $T$-psh function. Let $E \subset \mathcal{S}$ a bounded set, $x \in E$, and $r \in] 0,+\infty[$ such that $E \subset \subset B(x, r)$. Then,

$$
\sup _{E} u \leq \sup _{\partial B(x, r) \cap \mathcal{S}} u .
$$

In particular, $u$ does not have a strict local maximum.

Proof Let $x \in E$. By Lemma 1, we can find a pluri-Jensen measure $\mu_{x}$ with support in $\partial B(x, r) \cap \mathcal{S}$. Then, Remark 2 gives $u(x) \leq \int_{B(x, r) \cap \mathcal{S}} u \mathrm{~d} \mu_{x} \leq \sup _{\partial B(x, r) \cap \mathcal{S}} u$.

Note that, by virtue of Proposition 4.8.2 in [18], the previous lemma implies that the support of a closed positive $(1,1)$-current is nowhere pluri-thin, in the terminology of [18].

For a subset $A \in \mathbb{C}^{n}$ and a function $w: A \rightarrow \mathbb{R}$, we will denote $w^{*}$ its upper semicontinuous regularization, defined in $A$ by $w^{*}(x):=\lim \sup w(y)$.

We deduce from Lemma 1 the following result: $y \rightarrow x, y \in A$

Lemma 2 Let $T$ be a closed positive current defined in an open set $U \subset \mathbb{C}^{n}$. Let $\left(u_{n}\right)_{n}$ a locally uniformly bounded from above sequence of continuous co-T-psh functions. Then, the function $\sup ^{*} u_{n}$ is $T$-psh. 
Moreover, given an open set $W \subset \mathbb{C}^{n}$ and a holomorphic map $f: W \rightarrow U$, we have

$$
\left(\sup _{n}^{*} u_{n}\right) \circ f=\sup _{n}^{*}\left(u_{n} \circ f\right) .
$$

Proof Fix an open set $V \subset \subset U$. For each $n \in \mathbb{N}$, there exists a sequence $\left(v_{n, i}\right)_{i}$ of continuous plurisubharmonic functions defined in $V$, decreasing toward $u_{n}$ on $\mathcal{S} \cap V$. Thanks to Dini's Lemma, we may suppose $\left|v_{n, i}-u_{n}\right|<\frac{1}{n}$ on $\mathcal{S} \cap \bar{V}$ for all $n, i \in \mathbb{N}$. Then, the sequence of plurisubharmonic functions $\left(\sup ^{*} v_{n, i}\right)_{i}$ decreases on $\mathcal{S} \cap V$. (The upper regularization is done here in $V$.) We will prove that its limit on $S \cap V$ is precisely $\sup ^{*}\left(u_{n} \mid \mathcal{S} \cap V\right)$, which suffices to conclude.

For this purpose, it suffices to prove that $\forall i \in \mathbb{N}$, $\left.\sup _{n}^{*} v_{n, i}\right)\left.\right|_{\mathcal{S} \cap V}=\sup _{n}^{*}\left(v_{n, i \mid \mathcal{S} \cap V}\right)$. Suppose, by contradiction, that there exists $x_{0} \in \mathcal{S} \cap V$ and $i \in \mathbb{N}$ such that $\left.\left(\sup _{n}^{*} v_{n, i}\right)\right|_{\mathcal{S} \cap V}\left(x_{0}\right)>$ $\sup ^{*}\left(\left.v_{n, i}\right|_{\mathcal{S} \cap V}\right)\left(x_{0}\right)$. Then, by Proposition 4.8.2 in [18], there exists a plurisubharmonic function $v: V \rightarrow \mathbb{R}$ such that $\limsup _{x \rightarrow x_{0}, x \in \mathcal{S} \cap V \backslash\left\{x_{0}\right\}} v(x)<v\left(x_{0}\right)$. But this contradicts Corollary 1 applied to the $T$-psh function $\left.v\right|_{\mathcal{S} \cap V}: \mathcal{S} \cap V \rightarrow \mathbb{R}$.

An adaptation of the idea of the proof of Proposition 2.9.16 in [18] establishes the second statement, as follows. Denote by $J_{f}$ the Jacobian of $f$. For all $i \in \mathbb{N}$, the functions $\left(\sup _{n}^{*} u_{n, i}\right) \circ$ $f$ and $\sup ^{*}\left(u_{n, i} \circ f\right)$ (the upper regularization is done here in $W$ ) are two plurisubharmonic functions defined in $W$ which are equal in $W \backslash\left\{J_{f}=0\right\}$, hence everywhere. The previous argument shows that they decrease on $\mathcal{S} \cap W$ when $i \rightarrow+\infty$, respectively, toward the functions $\left(\sup ^{*} u_{n}\right) \circ f$ and $\sup ^{*}\left(u_{n} \circ f\right)$, which are therefore equal.

The following lemma gives an alternative definition for continuous $T$-psh functions.

Lemma 3 Let $T$ be a closed positive $(1,1)$-current with support $\mathcal{S}$ in an open set $U \subset \mathbb{C}^{n}$, and let $u: \mathcal{S} \rightarrow \mathbb{R}$ be a continuous function. Then, $u$ is $T$-psh if and only if $u(x) \leq \int_{U} u \mathrm{~d} \Lambda_{x}$, $\forall x \in \mathcal{S}$ and $\forall \Lambda_{x} \in P J_{\mathcal{S}}(x)$.

Proof Necessary condition. Consider a sequence $\left(v_{n}\right)_{n}$ of plurisubharmonic functions defined in an open set containing $\mathcal{S}$, such that $\left(v_{n}\right)_{n}$ decreases toward $u$ on $\mathcal{S}$. Let $x \in \mathcal{S}$ and $\Lambda_{x} \in P J_{\mathcal{S}}(x)$. The monotone convergence theorem gives

$$
v(x)=\lim _{n \rightarrow+\infty} v_{n}(x) \leq \lim _{n \rightarrow+\infty} \int_{U} v_{n} \mathrm{~d} \Lambda_{x}=\int_{U} v \mathrm{~d} \Lambda_{x} .
$$

Sufficient condition. Let $V \subset \subset U$ be an open set intersecting $\mathcal{S}$, and let $K:=\mathcal{S} \cap \bar{V}$. First let us recall an abstract duality theorem due to Edwards [9]. Let $Z$ be a compact metric space, and $\mathcal{R}$ a cone of continuous functions from $Z$ to $\mathbb{R}$, such that $\mathcal{R}$ separates points and contains the constants. Given $x \in Z$, we denote by $I_{x}$ the set of probability measures $\Lambda$ on $Z$ which satisfy $v(x) \leq \int_{K} v \mathrm{~d} \Lambda$ for all $v \in \mathcal{R}$. Let $\phi: Z \rightarrow \mathbb{R}$ be a continuous function. Then, Edwards' Theorem ensures that for all $x \in Z$,

$$
\sup \{v(x): v \in \mathcal{R}, v \leq \phi\}=\inf \left\{\int_{Z} \phi \mathrm{d} \Lambda: \Lambda \in I_{x}\right\} .
$$


Now let $u: \mathcal{S} \rightarrow \mathbb{R}$ be a continuous function such that $\forall x \in \mathcal{S}, \forall \Lambda_{x} \in P J_{\mathcal{S}}(x), u(x) \leq$ $\int_{U} u \mathrm{~d} \Lambda_{x}$. Let also $\left(A_{n}\right)_{n}$ be a compact exhaustion of $U$, and fix $m \in \mathbb{N}$. Denote $K_{m}:=$ $A_{m} \cap \mathcal{S}$. We apply Edwards' theorem with $\mathcal{R}$ being the set of continuous $K_{m}$-psh functions, $Z=K_{m}$ and $\phi=u$. Note that $I_{x}=P J_{K_{m}}(x)$. Indeed, (3) implies that $P J_{K_{m}}(x) \subset I_{x}$, while the inverse inclusion is trivial. We have $\forall x \in K_{m}$,

$$
\sup \{v(x) \mid v \in \mathcal{R}, v \leq u\} \leq u(x) \leq \inf \left\{\int_{K_{m}} u \mathrm{~d} \Lambda \mid \Lambda \in I_{x}\right\} .
$$

Then, Edwards' theorem gives $\forall x \in K_{m}$,

$$
u(x)=\sup \{v(x) \mid v \in R, v \leq u\}=\inf _{\Lambda_{x} \in P J_{K_{m}}(x)} \int_{K_{m}} u \mathrm{~d} \Lambda_{x} .
$$

We deduce that $u=u^{*}=\sup ^{*}\{v \mid v \in \mathcal{R}, v \leq u\}$ on $K_{m}$. But thanks to Lemma 2.3.4 in [18] and our Lemma $2, \sup ^{*}\{v \mid v \in \mathcal{R}, v \leq u\}$ is $T$-psh in $\stackrel{\circ}{K}_{m}$. The result follows by taking the limit $m \rightarrow+\infty$.

\subsection{Expression for relative Green's functions and applications}

Denote by $\Delta \subset \mathbb{C}$ the unit disk of $\mathbb{C}$. Let $U \subset \mathbb{C}^{n}$ be an open set. Recall that an analytic disk in $U$ with center $x \in U$ is a continuous function $h: \bar{\Delta} \rightarrow \mathbb{C}^{n}$, analytic in $\Delta$, such that $h(0)=x$. If $\lambda$ is the normalized Lebesgue measure on $\partial \Delta$, an analytic-disk measure on $U$ for $x$ is a measure of the form $g_{*} \lambda$, where $g: \bar{\Delta} \rightarrow U$ is an analytic disk in $U$ with center $x$.

We will denote by $\Lambda_{U}(x)$ the set of analytic disks which are pluri-Jensen measures relative to $x$ with support in an open set $U$. By Theorem 6.3 in [25], $P J_{U}(x)=\overline{\Lambda_{U}(x)}$.

We will need the following theorem of Poletsky (Corollary p. 170 in [20], see also [21]), generalized by Rosay [26]. Let $U$ be a connected complex manifold of dimension $\geq 1$. We denote by $\mathcal{H}_{z, U}$ the set of holomorphic functions $h: V_{h} \rightarrow U$ from some neighborhood $V_{h}$ of $\bar{\Delta}=\{z \in \mathbb{C},|z| \leq 1\}$ (possibly depending on $h$ ) into $U$ such that $h(0)=z$. We also denote by $P S H(U)$ the set of plurisubharmonic functions defined on $U$.

Theorem 1 Let $u: U \rightarrow \mathbb{R}$ be an upper semi-continuous function. With the previous notations, the function defined in $U$ by

$$
\tilde{u}(z):=\frac{1}{2 \pi} \inf _{f \in \mathcal{H}_{z, U}} \int_{0}^{2 \pi} u\left(f\left(e^{i \theta}\right)\right) \mathrm{d} \theta,
$$

if it is not everywhere equal to $-\infty$, belongs to $P S H(U)$ and verifies $\tilde{u} \leq u$. Moreover, this function $\tilde{u}$ is maximal among all the functions in $P S H(U)$ verifying this inequality. If $u$ is only assumed borelian, we still have $\tilde{u} \leq u$, and $\tilde{u}^{*}$ is plurisubharmonic.

The last statement in the above theorem is Lemma 2.2.12 in [28]. We prove below a version of this theorem, useful in our context (see also Corollary 1 in [24]). Let us recall some useful definitions.

An open bounded set $U \subset \mathbb{C}^{n}$ is called hyperconvex if there exists a continuous plurisubharmonic function $\rho: U \rightarrow]-\infty, 0[$ such that $\forall c \in]-\infty, 0\left[,\left\{z \in \mathbb{C}^{n}: \rho(z)<c\right\} \subset \subset U\right.$. In order to simplify the presentation, we will also suppose here that a hyperconvex set is connected. 
Let $U \subset \mathbb{C}^{n}, n \geq 1$, be a bounded open set, and let $A \subset \mathbb{C}^{n}$ be a compact set. The Green's function of $A \subset U$ relative to $U$ is defined by

$$
G_{A, U}:=\sup ^{*}\left\{v \in \operatorname{PSH}(U): v \leq 0,\left.v\right|_{A} \leq-1\right\},
$$

where sup* denotes the upper semi-continuous regularization of the upper envelope. A compact set $K \subset \mathbb{C}^{n}$ is called fat if $\overline{\stackrel{\circ}{K}}=K$. In this case, if $K$ is included into a bounded hyperconvex open set $U \subset \mathbb{C}^{n}$, the function $G_{K, U}$ is continuous by Proposition 4.5.3 of [18].

Theorem 2 Let $w: U \rightarrow \mathbb{R}$ be an upper semi-continuous function on a connected open set $U \subset \mathbb{C}^{n}$. Then, the function defined in $U$ by

$$
\hat{w}(x):=\inf _{\mu_{x} \in P J_{U}(x)} \int_{U} w \mathrm{~d} \mu_{x}
$$

is maximal among all the plurisubharmonic functions defined in $U$ and bounded from above by $w$ (if there are any).

In particular, if the open set $U$ is bounded and hyperconvex, and if $A \subset U$ is a fat compact set, the Green's function A relative to $U$ equals

$$
G_{A, U}(x)=\inf _{\mu_{x} \in P J_{U}(x)} \int_{U}\left(-\mathbf{1}_{A}\right)^{*} \mathrm{~d} \mu_{x}=-\sup _{\mu_{x} \in P J_{U}(x)} \mu_{x}(\AA) .
$$

In the case where the set $A \subset U$ is only assumed relatively compact, if the open set $U$ is bounded and hyperconvex, we have

$$
G_{A, U}(x) \geq \inf _{\mu_{x} \in P J_{U}(x)} \int_{U}\left(-\mathbf{1}_{\bar{A}}\right) \mathrm{d} \mu_{x} .
$$

Proof We denote again $\mathcal{H}_{z, U}$ the set of holomorphic functions $h: V_{h} \rightarrow U$ from a neighborhood $V_{h}$ of $\bar{\Delta}$ (possibly depending on $h$ ) into a neighborhood $U$ of $z \in \mathbb{C}^{n}$, such that $h(0)=z$.

Let $\mu_{x}=g_{*} \lambda$ with $g: \bar{\Delta} \rightarrow \mathbb{C}^{n}$ an analytic disk. By Mergelyan's Theorem, we can find a sequence $\left(f_{n}\right)_{n}$ of $\mathcal{H}_{z, U}$ which converges to $g$ uniformly on $\bar{\Delta}$. The upper semi-continuity of $w$ implies that $\forall \theta \in[0,2 \pi]$,

$$
\limsup _{n \rightarrow+\infty} w \circ f_{n}(\theta) \leq w \circ g(\theta) .
$$

It follows, by Fatou's Lemma, that:

$$
\limsup _{n \rightarrow+\infty} \frac{1}{2 \pi} \int_{0}^{2 \pi} w \circ f_{n}\left(e^{i \theta}\right) \mathrm{d} \theta \leq \int_{U} w \mu_{x} .
$$

(Note that this inequality also follows from Lemma 3.4.4 in [18].) We deduce that

$$
\frac{1}{2 \pi} \inf _{f \in \mathcal{H}_{z, U}} \int_{0}^{2 \pi} w \circ f\left(e^{i \theta}\right) \mathrm{d} \theta \leq \inf _{\nu_{x} \in \Lambda_{U}(x)} \int_{U} w \mathrm{~d} v_{x} .
$$

The inequality in the other direction being trivial, we finally have

$$
\inf _{v_{x} \in \Lambda_{U}(x)} \int_{U} w \mathrm{~d} v_{x}=\frac{1}{2 \pi} \inf _{f \in \mathcal{H}_{z, U}} \int_{0}^{2 \pi} w \circ f\left(e^{i \theta}\right) \mathrm{d} \theta .
$$

On the other hand, recall that every pluri-Jensen measure is a limit of analytic disks. For $v_{x} \in P J_{U}(x)$, denote $\left(v_{x, n}\right)_{n}$ a sequence of analytic disks, weakly convergent to $v_{x}$. Thanks 
to the upper semi-continuity of $w$ (see, e.g., Lemma 2.8 in [4], or Lemma 3.4.4 in [18]), we have $\forall v_{x} \in P J_{U}(x)$,

$$
\inf _{\mu_{x} \in \Lambda_{U}(x)} \int_{U} w \mathrm{~d} \mu_{x} \leq \lim _{n \rightarrow+\infty} \int_{U} w \mathrm{~d} v_{x, n} \leq \int_{U} w \mathrm{~d} v_{x} .
$$

Bringing together equations (7) and (8), we obtain

$$
\begin{aligned}
\inf _{v_{x} \in P J_{U}(x)} \int_{U} w \mathrm{~d} v_{x} & \geq \inf _{v_{x} \in \Lambda_{U}(x)} \int_{U} w \mathrm{~d} v_{x}=\frac{1}{2 \pi} \inf _{f \in \mathcal{H}_{z, U}} \int_{0}^{2 \pi} w \circ f\left(e^{i \theta}\right) \mathrm{d} \theta \\
& \geq \inf _{v_{x} \in P J_{U}(x)} \int_{U} w \mathrm{~d} v_{x},
\end{aligned}
$$

and hence the above inequalities are all equalities. Theorem 1 concludes the proof of (4).

Recall that $G_{A, U}$ is continuous by Proposition 4.5.3 of [18]. Then, (5) comes from (4), since

$$
G_{A, U}(x)=\inf _{\mu_{x} \in P J_{U}(x)} \int_{U}\left(-\mathbf{1}_{\AA}\right) \mathrm{d} \mu_{x} \geq \inf _{\mu_{x} \in P J_{U}(x)} \int_{U}\left(G_{A, U}\right) \mathrm{d} \mu_{x}=G_{A, U}(x) .
$$

Let us now show (6). Denote by $\operatorname{dist}(\cdot, \cdot)$ the euclidian distance. Take $\epsilon>0$ such that $U_{\epsilon}:=\{z \in U, \operatorname{dist}(z, \partial U)<\epsilon\}$ is non-empty and contains $A$. Take $x \in U_{\epsilon}$. Let $A_{m}:=$ $\left\{z \in \mathbb{C}^{n}, \operatorname{dist}(z, A) \leq \frac{1}{m}\right\}$ for every $m \in \mathbb{N}^{*}$ sufficiently large such that $A_{m} \subset \subset U_{\epsilon}$. Let then $\left(v_{x, n, k}\right)_{k}$ be a weakly convergent sequence of measures in $P J_{U_{\epsilon}}(x)$ such that

$$
\inf _{v_{x} \in J_{U_{\epsilon}}(x)} \int_{U_{\epsilon}}\left(-\mathbf{1}_{A_{n}}\right) \mathrm{d} v_{x}=\lim _{k \rightarrow+\infty} \int_{U_{\epsilon}}\left(-\mathbf{1}_{A_{n}}\right) \mathrm{d} v_{x, k, n} .
$$

Denote $v_{x, n}^{\prime}$ its limit, and note that $v_{x, n}^{\prime} \in P J_{\overline{U_{\epsilon}}}(x)$ (see Theorem 6.3 in [25]).

Let also $v_{x}^{\prime} \in P J_{\bar{U}_{\epsilon}}(x)$ be an accumulation point of the sequence $\left(v_{x, n}^{\prime}\right)_{n}$, toward which we may suppose that it converges. Note that the sequence $\left(-\mathbf{1}_{A_{n}}\right)_{n}$ is a non-decreasing sequence of lower semi-continuous functions. Lemma 2.8 in [4] used twice implies that

$$
\begin{aligned}
G_{A, U_{\epsilon}}(x) & \geq \lim _{n \rightarrow+\infty} G_{A_{n}, U_{\epsilon}}(z)=\lim _{n \rightarrow+\infty} \lim _{k \rightarrow+\infty} \int_{U_{\epsilon}}\left(-\mathbf{1}_{A_{n}}\right)^{*} \mathrm{~d} v_{x, n, k} \\
& \geq \limsup _{n \rightarrow+\infty} \limsup _{k \rightarrow+\infty} \int_{U_{\epsilon}}\left(-\mathbf{1}_{A_{n}}\right) \mathrm{d} v_{x, n, k} \geq \limsup _{n \rightarrow+\infty} \int_{\overline{U_{\epsilon}}}\left(-\mathbf{1}_{A_{n}}\right) \mathrm{d} v_{x, n}^{\prime} \\
& \geq \int_{\overline{U_{\epsilon}}}\left(-\mathbf{1}_{\bar{A}}\right) \mathrm{d} v_{x}^{\prime} \geq \inf _{\mu_{x} \in J_{\overline{U_{\epsilon}}}}(x) \int_{\overline{U_{\epsilon}}}\left(-\mathbf{1}_{\bar{A}}\right) \mathrm{d} \mu_{x} \geq \inf _{\mu_{x} \in P J_{U}(x)} \int_{U}\left(-\mathbf{1}_{\bar{A}}\right) \mathrm{d} \mu_{x} .
\end{aligned}
$$

Now, since $U$ is assumed hyperconvex, Proposition 4.5.7 in [18] allows us to pass to the limit

$$
G_{A, U}(x)=\lim _{\epsilon \rightarrow 0} G_{A, U_{\epsilon}}(x) \geq \inf _{\mu_{x} \in P J_{U}(x)} \int_{U}\left(-\mathbf{1}_{\bar{A}}\right) \mathrm{d} \mu_{x} .
$$

Remark 3 It follows from Theorem 2 that for a bounded hyperconvex open set $U \subset \mathbb{C}^{n}$, if $A \subset$ $U$ is a fat compact set such that $\hat{A} \subset U$, then $\forall x \in U, \sup _{v_{x} \in P J_{U}(x)} v_{x}(\AA)=\sup _{v_{x} \in P J_{U}(x)} v_{x}(\dot{\hat{A}})$. Indeed, $\forall x \in U$, we have

$$
\inf _{v_{x} \in P J_{U}(x)} \int_{U}\left(-\mathbf{1}_{A}\right)^{*} \mathrm{~d} v_{x}=G_{A, U}(x)=G_{\hat{A}, U}(x)=\inf _{v_{x} \in P J_{U}(x)} \int_{U}\left(-\mathbf{1}_{\hat{A}}\right)^{*} \mathrm{~d} v_{x} .
$$


(The equality $G_{A, U}=G_{\hat{A}, U}$ comes from Theorem 5.1.7 in [18] and Corollary 5.3.4 in [18].) Thus, $A$ is polynomially convex if and only if $\forall x \notin A$,

$$
\sup _{v_{x} \in P J_{U}(x)} v_{x}(\AA)<1 .
$$

Finally, since $P J_{\bar{U}}(x)$ is closed (Lemma 7.2 in [17], see also Theorem 6.3 in [25]), Lemma 3.4.4 in [18] implies

$$
A=\hat{A} \Longleftrightarrow \forall x \notin A, \forall v_{x} \in P J_{U}(x), v_{x}(\AA)<1 .
$$

Application 1 In particular, Remark 3 allows to deduce that, if $u: \mathbb{C}^{n} \rightarrow \mathbb{R}^{+}$is a continuous plurisubharmonic function, then the set $\{u=0\}$ is polynomially convex, provided that it is bounded and fat.

Indeed, suppose that $\{u=0\}$ is bounded and fat but not polynomially convex. By Remark 3 , one can find $y \in \mathbb{C}^{n} \backslash\{u=0\}$ and a measure $\mu_{y} \in P J_{y}\left(\mathbb{C}^{n}\right)$ whose support is included in $\{u=0\}$. Then,

$$
0<u(y) \leq \int_{\mathbb{C}^{n}} u \mathrm{~d} \mu_{y} \leq \int_{\mathbb{C}^{n}} 0 \mathrm{~d} \mu_{y}=0 .
$$

We have thus proved by contradiction that $\{u=0\}$ is polynomially convex, if it is bounded and fat.

Corollary 2 Let $U \subset \mathbb{C}^{n}$ be an open set, and let $A \subset U$ be a complete pluripolar set, i.e., $A=\{u=-\infty\}$ for a plurisubharmonic function $u: U \rightarrow \mathbb{R} \cup\{-\infty\}$. Then, $\forall x \in U \backslash A$, the set $A$ is of null measure for every pluri-Jensen measure in $P J_{U}(x)$.

Conversely, suppose $U$ bounded and hyperconvex. If there exists $x \in U \backslash \bar{A}$ such that the set $A$ is of null measure for every pluri-Jensen measure in $P J_{U}(x)$, then $A$ is pluripolar.

Proof Let $x \in \mathbb{C}^{n} \backslash A$, and $\mu_{x} \in P J_{U}(x)$. We have $\mu_{x}(A)=0$, since

$$
-\infty<u(x) \leq \int_{U} u \mathrm{~d} \mu_{x} .
$$

Conversely, we may suppose that $A$ is compact. Suppose $\exists x \in U \backslash \bar{A}, \forall v_{x} \in P J_{U}(x)$, we have $v_{x}(A)=0$. Then, Theorem 1 implies that

$$
0=\inf _{v_{x} \in P J_{U}(x)} \int_{U}\left(-\mathbf{1}_{\bar{A}}\right) \mathrm{d} v_{x} \leq G_{A, U}(x) .
$$

The function $G_{A, U}$ is null in a point outside $\bar{A}$, hence everywhere on $U$, and thus $A$ is pluripolar.

Remark 4 Note that, given a borelian function $u: U \rightarrow \mathbb{R} \cup\{-\infty\}$ such that the set $\{u=$ $-\infty\}$ is complete pluripolar, we deduce, by applying Theorem 1 to $U \backslash\{u=-\infty\}$, that the statement of Theorem 1 remains valid when replacing $u: U \rightarrow \mathbb{R}$ by $u: U \rightarrow \mathbb{R} \cup\{-\infty\}$. Similarly, the statement of Theorem 2 remains valid when replacing $w: U \rightarrow \mathbb{R}$ by $w:$ $U \rightarrow \mathbb{R} \cup\{-\infty\}$.

We obtain the following lemma, which we will often use in the sequel:

Lemma 4 Let $T$ be a closed positive $(1,1)$-current defined in a connected open set $U \subset \mathbb{C}^{n}$. Let $v: \mathcal{S} \rightarrow \mathbb{R}^{-}$be a $T$-psh function. If $u: U \rightarrow \mathbb{R}^{-}$is the extension of $v$ to $U$ with the value 0 , we have $\forall x \in \mathcal{S}$,

$$
\inf _{v_{x} \in P J_{U}(x)} \int_{U} u \mathrm{~d} \mu_{x}=\inf _{v_{x} \in P J_{\mathcal{S}}(x)} \int_{\mathcal{S}} v \mathrm{~d} \mu_{x}=v(x) .
$$


Proof First suppose that $v=\left.w\right|_{\mathcal{S}}$ for some plurisubharmonic function $w: U \rightarrow \mathbb{R}$ such that $\left.w\right|_{\mathcal{S}}<0$. Denote by $W$ the open set $\{w<0\} \subset U$, and note that $\mathcal{S} \subset W$. Then, Theorem 2 and (2) imply that $\forall x \in \mathcal{S}$ we have

$$
\begin{aligned}
v(x) & =\inf _{v_{x} \in P J_{U}(x)} \int_{U} w \mathrm{~d} v_{x} \leq \inf _{v_{x} \in P J_{W}(x)} \int_{U} w \mathrm{~d} v_{x} \\
& \leq \inf _{v_{x} \in P J_{W}(x)} \int_{U} u \mathrm{~d} v_{x} \leq \inf _{v_{x} \in P J_{\mathcal{S}}(x)} \int_{U} u \mathrm{~d} v_{x}=v(x) .
\end{aligned}
$$

Now suppose only that $v: \mathcal{S} \rightarrow \mathbb{R}^{-}$is a $T$-psh function as in the statement. Let $\left(v_{n}\right)_{n}$ be a sequence of plurisubharmonic functions defined in $U$, decreasing on $\mathcal{S}$ toward $v$. Take $x \in \mathcal{S}$ and $\mu_{x} \in P J_{U}(x)$. Take $\epsilon>0$. Then, there exists $n_{0}$ such that $v_{n}(x)-\epsilon<0$ for $n \geq n_{0}$. There also exists an open neighborhood $O \subset U$ of $x$ such that $O \cap \mathcal{S} \subset \bigcap_{n \geq n_{0}}\left\{v_{n}-\epsilon<0\right\}$. Up to increasing $n_{0}$, we may suppose that the compact support of $\mu_{x}$ is included in $O$. Denote by $u_{n, \epsilon}$ the extension to $U$ with the value 0 of $\left.\left(v_{n}-\epsilon\right)\right|_{\mathcal{S}}$, and denote by $u_{n}$ the extension to $U$ with the value 0 of $\left.v_{n}\right|_{\mathcal{S}}$. By the previous step,

$$
v_{n}(x)-\epsilon=\inf _{v_{x} \in P J_{O}(x)} \int_{U} u_{n, \epsilon}(x) v_{x} \leq \int_{U} u_{n} \mathrm{~d} \mu_{x} .
$$

The monotone convergence theorem and the arbitrary choice of $\epsilon>0$ give $v(x) \leq \int_{U} u \mathrm{~d} \mu_{x}$. Then, $v(x)=\inf _{\mu_{x} \in P J_{U}(x)} \int_{U} u \mathrm{~d} \mu_{x}$ since $\delta_{x} \in P J_{U}(x)$.

Let us now introduce a new notion, which we will use in the next result.

Definition 2 Let $\mathcal{S}$ be the support of a closed positive $(1,1)$-current $T$. A set $E \subset \mathcal{S}$ is called $T$-pluripolar if there exists a $T$-psh function $v: \mathcal{S} \rightarrow \mathbb{R} \cup\{-\infty\}$ such that $e^{u}$ is continuous and $E \subset\{v=-\infty\}$.

We prove the following result.

\section{Proposition 1 A set $F \subset S$ which is T-pluripolar is pluripolar.}

Proof Without loss of generality, we may suppose that $F$ is bounded, since a locally pluripolar set is pluripolar. Let $u: \mathcal{S} \rightarrow \mathbb{R} \cup\{-\infty\}$ be a $T$-psh function with $e^{u}$ continuous, and $E=\{u=-\infty\} \subset \mathcal{S}$ a $T$-pluripolar set containing $F$. Let $U \subset \mathbb{C}^{n}$ be an open bounded hyperconvex set containing $F$, and let $\left(u_{n}\right)_{n}$ be a sequence of continuous plurisubharmonic functions defined in $U$, decreasing toward $u$ on $\mathcal{S}$. Let $v_{n}:=\left.u_{n}\right|_{\mathcal{S}}$ extended to $U$ with the value 0 . Let $K \subset U \cap \mathcal{S}$ be a non-pluripolar compact set containing also $F$. Up to shrinking $U$, we may suppose that $u_{n} \leq 0$ on $U \cap \mathcal{S}$ for $n \geq n_{0}$. Theorem 2 and Lemma 4 imply that $\forall n \geq n_{0}, \forall c \in \mathbb{R}^{-} \backslash\{0\}$, we have $\forall x \in U \cap \mathcal{S}$,

$$
\begin{aligned}
& G_{\left\{u_{n} \leq c\right\} \cap K, U}(x) \geq \inf _{\mu_{x} \in P J_{U}(x)} \int_{U}\left(-\mathbf{1}_{\left\{u_{n} \leq c\right\} \cap K}\right) \mathrm{d} \mu_{x} \\
& \geq \inf _{\mu_{x} \in P J_{U}(x)} \int_{\left\{u_{n} \leq c\right\} \cap \mathcal{S}}\left(-\mathbf{1}_{\left\{u_{n} \leq c\right\}}\right) \mathrm{d} \mu_{x} \\
& \geq \frac{1}{|c|} \inf _{\mu_{x} \in P J_{U}(x)} \int_{\left\{u_{n} \leq c\right\} \cap \mathcal{S}} v_{n} \mathrm{~d} \mu_{x} \geq \frac{1}{|c|} \inf _{\mu_{x} \in P J_{U}(x)} \int_{U \cap \mathcal{S}} v_{n} \mathrm{~d} \mu_{x}=\frac{u_{n}(x)}{|c|} .
\end{aligned}
$$

Corollary 4.7.8 in [18] allows us to pass to the limit : $\forall c \in \mathbb{R}^{-} \backslash\{0\}, \forall x \in U \cap \mathcal{S}$,

$$
G_{\{u \leq c\} \cap K, U}(y)=\lim _{n \rightarrow+\infty} G_{\left\{u_{n} \leq c\right\} \cap K, U}(y) \geq \frac{u(x)}{|c|} .
$$


Now let $y \in(U \cap \mathcal{S}) \backslash E$. We have

$$
G_{E \cap K, U}(y) \geq \lim _{c \rightarrow-\infty} G_{\{u \leq c\} \cap K, U}(y) \geq 0 .
$$

Since we obtain $G_{E \cap K, U}=0$ on $(U \cap \mathcal{S}) \backslash E$, hence on $U$, it follows that the set $E \cap K$ is pluripolar. Thus, $F \subset E \cap K$ is pluripolar.

Remark 5 It is not always true that a pluripolar set is $T$-pluripolar: for instance, $B(0,1) \cap$ $\left\{z_{1}=0\right\}$ is pluripolar in $\mathbb{C}^{2}=\left\{\left(z_{1}, z_{2}\right): z_{1}, z_{2} \in \mathbb{C}\right\}$. This set is not $T$-pluripolar for $T=d d^{c} \log \left|z_{1}\right|$, because $\left(z_{1}, z_{2}\right) \mapsto \log \left|z_{1}\right| \notin L_{\text {loc }}^{1}(\|T\|)$.

Using Proposition 1, the $T$-pluripolar sets are the pluripolar subsets of $\operatorname{supp}(T)$ which are included in a set of the form $\{u=-\infty\}$, for a continuous plurisubharmonic function $u \in$ $L_{\text {loc }}^{1}(\|T\|)$. This is the case, for example, if the set $\{u=-\infty\} \cap \operatorname{supp}(T)$ is relatively compact in a ball contained in the domain of definition of $u$, by Proposition 3.1 in [4], or if it is of Hausdorff measure $\mathcal{H}_{1}(E)=0$, by Theorem 3.5 in [4].

We will further need the following lemma:

Lemma 5 Let $U \subset \mathbb{C}^{n}$ be an open bounded hyperconvex set, and $A \subset \subset U$ a non-pluripolar fat compact set. Then, for every $\epsilon>0$, there exists a neighborhood $V \subset \subset U$ of A such that for every $x \in V$, there exists a pluri-Jensen measure $v_{x} \in P J_{U}(x)$ with $\nu_{x}(A) \geq 1-\epsilon$.

Proof By Theorem 2,

$$
\inf _{v_{x} \in P J_{U}(x)} \int_{U}\left(-\mathbf{1}_{A}\right) \mathrm{d} v_{x} \leq G_{A, U}(x) .
$$

It suffices thus to take $V:=\left\{G_{A, U}<-1+\frac{\epsilon}{2}\right\}$.

\subsection{Properties analogous to the ones for plurisubharmonic functions}

We prove here several results which transpose to $T$-psh functions certain properties of plurisubharmonic functions.

Lemma 6 Let $T$ be a closed positive current in an open subset of $\mathbb{C}^{n}$. Let $\left(u_{m}\right)_{m}$ be a locally uniformly bounded from above sequence of continuous co-T-psh functions. Suppose that the upper semi-continuous regularization $u_{\infty}^{*}: \mathcal{S} \rightarrow \mathbb{R}$ of the function $u_{\infty}:=\limsup _{m \rightarrow+\infty} u_{m}$ is continuous. Then, the set $\left\{u_{\infty}<u_{\infty}^{*}\right\}$ is pluripolar.

Proof First suppose that $u_{\infty}^{*}$ is constant. Up to replacing $u_{m}$ by $u_{m}-u_{\infty}^{*}$, we may suppose that $u_{\infty}^{*}=0$. Let $V$ be an open hyperconvex subset of $\mathbb{C}^{n}$ intersecting $\mathcal{S}$, and $K \subset V \cap \mathcal{S}$ a non-pluripolar compact set. Let a constant $\epsilon>0$. For every $m \in \mathbb{N}$, by Dini's Lemma, up to shrinking $V$, there exists a continuous plurisubharmonic function $v_{m}: V \rightarrow \mathbb{R}$, with $v_{m} \geq u_{m}$ on $\mathcal{S}$ and $\left|v_{m}-u_{m}\right| \leq \frac{1}{m}$ on $\mathcal{S}$. We can also suppose that for every $m \in \mathbb{N}$ we have $v_{m}<\epsilon$ on $\mathcal{S} \cap V$. Then, $u_{\infty}=\left.\limsup _{m \rightarrow+\infty} v_{m}\right|_{\mathcal{S}}$ on $V \cap \mathcal{S}$.

Let a constant $c<-\epsilon$. Let $x \in V \cap \mathcal{S} \backslash\left\{u_{\infty}<0\right\}$. There exists an extracted subsequence $\left(v_{m_{j}}\right)_{j}$ of $\left(v_{m}\right)_{m}$ such that $\left(v_{m_{j}}(x)\right)_{j}$ converges to 0 . Then, $\forall j \in \mathbb{N}$, a computation similar to the one in the proof of Proposition 1 gives $\forall j \in \mathbb{N}$,

$$
G_{\left\{\sup _{m \geq m_{j}} v_{m}-\epsilon \leq c\right\} \cap K, V}(x) \geq G_{\left\{v_{m_{j}}-\epsilon \leq c\right\} \cap K, V}(x) \geq \frac{v_{m_{j}}(x)-\epsilon}{|c|} .
$$


Corollary 4.7.8 in [18] allows us to pass to the limit

$$
G_{\left\{u_{\infty}-\epsilon \leq c\right\} \cap K, V}(x)=\lim _{j \rightarrow+\infty} G_{\left\{\sup _{m \geq m_{j}} v_{m}-\epsilon \leq c\right\} \cap K, V}(x) \geq \lim _{j \rightarrow+\infty} \frac{v_{m_{j}}(x)-\epsilon}{|c|}=\frac{-\epsilon}{|c|} .
$$

Corollary 4.7.8 in [18] allows us to take the limit $\epsilon \rightarrow 0$, and to obtain

$$
G_{\left\{u_{\infty} \leq c\right\} \cap K, V}(x)=0 .
$$

The function $G_{\left\{u_{\infty} \leq c\right\} \cap K, V}$ is null at $x$, hence on $V$. Finally, Corollary 4.7.8 in [18] allows again to pass to the limit

$$
G_{\left\{u_{\infty}<0\right\} \cap K, V}=\lim _{c \rightarrow 0^{-}} G_{\left\{u_{\infty} \leq c\right\} \cap K, V}=0 .
$$

Thus, by the arbitrary choice of $K$, the set $\left\{u_{\infty}<0\right\}$ is pluripolar.

Now we suppose only that $u_{\infty}^{*}$ is continuous. Remark that in this case the previous reasoning shows that for every compact set $K \subset \mathcal{S}$ and every real number $c<u_{\infty}^{*}$ on $K$, the set $\left\{u_{\infty}<c\right\} \cap K$ is pluripolar. Indeed, under these conditions, (9) is still true.

Let $\left(s_{m}\right)_{m}$ be an increasing sequence of step functions, locally uniformly convergent toward $u_{\infty}^{*}$. By the previous remark, for all $m \in \mathbb{N}$, the set $\left\{\phi_{\infty}<s_{m}\right\}$ is pluripolar. Thus, the set $\left\{\phi_{\infty}<\phi_{\infty}^{*}\right\}=\bigcup_{m \in \mathbb{N}}\left\{\phi_{\infty}<s_{m}\right\}$ is pluripolar.

We further present a version of Hartogs's Theorem for $T$-psh functions.

Theorem 3 Let $\mathcal{S}$ be the support of a closed positive $(1,1)$-current $T$ in an open bounded set $U \subset \mathbb{C}^{n}$. Let also $Q \subset \mathbb{C}^{n}$ be a fat compact set such that $K:=Q \cap \mathcal{S}$ is non-pluripolar, and $E \subset K$ a set whose closure $\bar{E}$ in $K$ is of empty interior for the induced topology. Let $\left(u_{n}\right)_{n}: U \cap \mathcal{S} \rightarrow \mathbb{R}$ be a sequence of continuous co-T-psh functions uniformly bounded from above on $U$, such that $\lim _{n \rightarrow+\infty}\left(\sup _{i \geq n} u_{i}\right)^{*} \leq f$ on $K \backslash E$ for some continuous function $f: \mathcal{S} \cap U \rightarrow \mathbb{R}$. Then, for every $\epsilon>0$, there exists $n_{0} \in \mathbb{N}$, and a neighborhood $V \subset U$ of $K$ such that $u_{n} \leq f+\epsilon$ on $V \cap \mathcal{S}$ for all $n \geq n_{0}$. In particular, $\lim \sup u_{n} \leq C$ on $K$.

Proof First suppose that $f$ is equal to a constant $C$. We may suppose, up to subtracting a constant, that $u_{n}<0$ in $U \cap \mathcal{S}$ and that $C<0$. Let us denote $u_{n}^{\prime}:=\left(\sup _{i \geq n} u_{i}\right)^{*}$. Then, the sequence of upper semi-continuous functions $\left(u_{n}^{\prime}\right)_{n}$ decreases on $\mathcal{S}$ toward $u:=\lim _{n \rightarrow+\infty}\left(\sup _{i \geq n} u_{i}\right)^{*}$. By Lemma 5, there exists a neighborhood $V$ of $K$ such that $\forall x \in V \cap \mathcal{S}$, we can find $v_{x} \in P J_{U}(x)$ such that $v_{x}(K)>1-\frac{\epsilon}{2|C+\epsilon|}$.

By Egoroff's Theorem, there exists a set $A \subset K$, with $v_{x}(A)<\frac{\epsilon}{2|C+\epsilon|}$, such that $\left(u_{n}^{\prime}\right)_{n}$ converges uniformly toward $u$ on $K \backslash A$. Fix $\epsilon>0$ such that $C+2 \epsilon<0$. Then, $\exists n_{0} \in \mathbb{N}$ such that $\forall z \in K \backslash(A \cup E), \forall n \geq n_{0}: u_{n}(z) \leq u_{n}^{\prime}(z) \leq C+\epsilon$. The continuity of the functions $u_{n}$ then implies that $\forall z \in K \backslash A, \forall n \geq n_{0}$ we have

$$
u_{n}(z) \leq C+\epsilon \text {. }
$$

Extend each $u_{n}$ to $U$ with the value 0 . By the negativity of the functions $u_{n}$ on $\mathcal{S} \cap U$, we have for $n \geq n_{0}$ and $x \in V \cap \mathcal{S}$, thanks to (2) and Lemma 4, 


$$
\begin{aligned}
u_{n}(x) & =\inf _{\mu_{x} \in P J_{U \cap \mathcal{S}}(x)} \int_{U} u_{n} \mathrm{~d} \mu_{x}=\inf _{\mu_{x} \in P J_{U}(x)} \int_{U} u_{n} \mathrm{~d} \mu_{x} \leq \int_{U} u_{n} \mathrm{~d} v_{x} \\
& \leq \int_{K \backslash A} u_{n} \mathrm{~d} v_{x} \leq v_{x}(K \backslash A) \sup _{K \backslash A} u_{n} \leq\left(1-\frac{\epsilon}{|C+\epsilon|}\right)(C+\epsilon)=C+2 \epsilon .
\end{aligned}
$$

Now suppose that $f$ is a continuous function. Let $\left(s_{m}\right)_{m}$ be a sequence of step functions decreasing toward $f$, uniformly on $K$. There exists $m_{0} \in \mathbb{N}$ such that $s_{m_{0}}-f \leq \epsilon$. The previous step implies that, for $m$ sufficiently large we have on $K$ :

$$
u_{m} \leq s_{m_{0}} \leq f+\epsilon,
$$

which proves the stated result.

We deduce the following result:

Proposition 2 Let $\mathcal{S}$ be the support, supposed non-pluripolar, of a closed positive (1,1)current $T$ in an open set $U \subset \mathbb{C}^{n}$. A $T$-psh function $u: \mathcal{S} \rightarrow \mathbb{R}$, constant outside a set $E$ whose closure in $\mathcal{S}$ is of empty interior, is constant everywhere.

Proof Let us suppose that $u=C$ outside $E$. The upper semi-continuity of $u$ implies that $E=\{u \neq C\}=\{u>C\}$. Let $\left(u_{n}\right)_{n}$ be a sequence of continuous plurisubharmonic functions decreasing on $\mathcal{S}$ toward $u$. Theorem 3 applied to $\left(u_{n}\right)_{n}$ on compact subsets of $\mathcal{S}$ concludes.

This result allows us to weaken the conditions required in the statement of Theorem 3:

Corollary 3 In Theorem 3, if the current $T$ does not charge pluripolar sets, we can replace the condition $\lim _{n \rightarrow+\infty}\left(\sup _{i \geq n} u_{i}\right)^{*} \leq f$ by the less restrictive condition $\lim _{n \rightarrow+\infty}\left(\sup _{i \geq n} u_{i}\right) \leq f$.

Proof We use the same notations as those in the statement of Theorem 3. As in the proof of this theorem, we can suppose that the continuous function $f$ is equal to a constant $C \in \mathbb{R}$. It suffices to show that

$$
\lim _{n \rightarrow+\infty} \sup _{i \geq n} u_{i} \leq C \Rightarrow \lim _{n \rightarrow+\infty}\left(\sup _{i \geq n} u_{i}\right)^{*} \leq C .
$$

It is sufficient again, by Lemma 2 and Proposition 2, to show that $\lim _{n \rightarrow+\infty} \sup _{i \geq n} u_{i} \leq C$ implies that $\lim _{n \rightarrow+\infty} \max \left(\left(\sup _{i \geq n} u_{i}\right)^{*}, C\right)=C$ on $\mathcal{S} \backslash E$, where $E \subset \mathcal{S}$ is a closed set of empty interior for the induced topology. Suppose that the last condition is false. By Baire's theorem, there exists $k \in \mathbb{N} \backslash\{0\}$ such that

$$
\lim _{n \rightarrow+\infty}\left(\sup _{i \geq n} u_{i}\right)^{*} \geq C+\frac{1}{k}
$$

in an open set (for the induced topology) $W \subset \mathcal{S}$. Then, $\left(\sup _{i \geq m} u_{i}\right)^{*} \geq C+\frac{1}{k}$ in $W, \forall m \in \mathbb{N}$. Hence there exists a non-empty set $O \subset W$ dense in $W$ (for the induced topology) such that $\forall m \in \mathbb{N}, \sup _{i \geq m} u_{i} \geq C+\frac{1}{2 k}$ on $O$. This implies that $\lim _{n \rightarrow+\infty} \sup _{i \geq n} u_{n}>C$ on $O$, which finishes the proof. 
We can now state a result which improves Corollary 1 for certain currents:

Proposition 3 Let $T$ be a closed positive $(1,1)$-current in an open set $U \subset \mathbb{C}^{n}$, with support $\mathcal{S} \subset U$. Let $\left(v_{m}\right)_{m}$ be a sequence of $T$-psh functions, such that $\lim \sup v_{m} \leq C$ for some $C \in \mathbb{R}$. If $\lim _{m \rightarrow+\infty} v_{m}(x)=C$ for some $x \in \mathcal{S}$, then $\left(v_{m}\right)_{m}$ converges toward $C$ outside a pluripolar set in $\mathcal{S}$.

In particular, if $\|T\|$ does not charge pluripolar sets, then any $T$-psh function that reaches its maximum is constant.

Proof Let us suppose $C=0$. Note that the function $\limsup _{m \rightarrow+\infty}^{*} v_{m}: \mathcal{S} \rightarrow \mathbb{R}$ is $T$-psh by Lemma 2. Extend each function $v_{m}$ to $U$ with the value 0. Fatou's lemma and Lemma 4 imply that $\forall v_{x} \in P J_{U}(x)$,

$$
0=\lim _{m \rightarrow+\infty} v_{m}(x) \leq \liminf _{m \rightarrow+\infty} \int_{U} v_{m} \mathrm{~d} v_{x} \leq \limsup _{m \rightarrow+\infty} \int_{U} v_{m} \mathrm{~d} v_{x} \leq \int_{U} \limsup _{m \rightarrow+\infty}^{*} v_{m} \mathrm{~d} v_{x} \leq 0 .
$$

Finally $\forall v_{x} \in P J_{U}(x), \lim _{m \rightarrow+\infty} \int_{U} v_{m} \mathrm{~d} v_{x}=0$. Thus, $\left(v_{m}\right)_{m}$ extended to $U$ with the value 0 converges $v_{x}$-a.e. toward 0 on the support of every measure $v_{x} \in P J_{U}(x)$. Since $P J_{U}(x)$ is invariant by rotations and homotheties with center $x$, Lemma 1 and Corollary 2 imply that $\left(v_{n}\right)_{n}$ converges toward 0 on $\mathcal{S} \backslash E$, where $E \subset \mathcal{S}$ is a pluripolar set. In particular, if $\|T\|$ does not charge pluripolar sets, then any $T$-psh function that reaches its maximum is constant outside such a set $E$, hence everywhere by Proposition 2 .

Finally, let us prove an inequality of Chern-Levine-Nirenberg type for $T$-psh functions:

Proposition 4 Let $r_{0}>0$ be a real number. If $u$ is a continuous $T$-psh function, then there exists a constant $c \geq 0$ such that $\forall r<\frac{r_{0}}{2}$,

$$
T \wedge d d^{c} u(B(z, r)) \leq \frac{c}{r^{2 n}}\|T\|(B(z, 2 r)) \sup _{\operatorname{supp}(T) \cap B(z, 2 r)} u .
$$

Proof It suffices to prove the above formula when $u$ is the restriction of a plurisubharmonic function to the support $\mathcal{S}$ of $T$. The monotone convergence theorem then allows us to extend the formula to the general case.

Let $\xi$ be a positive step function equal to 1 in $B(z, 1)$ and to 0 in $B(z, 2)$. Let us denote $\xi_{r}(z):=\xi\left(\frac{z}{r}\right)$. Then, $\left.\forall r \in\right] 0,1[$,

$$
\begin{aligned}
T \wedge d d^{c} u(B(z, r)) & \leq \int_{\mathbb{C}^{n}} \xi_{r} T \wedge d d^{c} u=\int_{\mathbb{C}^{n}} u T \wedge d d^{c} \xi_{r} \\
& \leq \frac{c}{r^{2 n}}\|T\|(B(z, 2 r)) \sup _{\mathcal{S} \cap B(z, 2 r)} u .
\end{aligned}
$$

\section{Applications to dynamics in $\mathbb{C}^{n}$}

\subsection{Background}

Given a polynomial application $f: \mathbb{C}^{n} \rightarrow \mathbb{C}^{n}$, we extend it to a meromorphic endomorphism $\left[P_{1}, \ldots, P_{n}\right]: \mathbb{P}^{n} \rightarrow \mathbb{P}^{n}$, where $P_{1}, \ldots, P_{n}$ are homogeneous polynomials. The choice of 
$\mathbb{P}^{n}$ as compactification of $\mathbb{C}^{n}$ is practical in our case because the group $H^{1,1}\left(\mathbb{P}^{n}, \mathbb{R}\right)$ is of dimension 1, and the classes which compose it are Kählerian, in the sense that they contain a strictly positive smooth $(1,1)$-form. Denote by $H \subset \mathbb{P}^{n}$ the hyperplane at infinity. Denote by $I_{f} \subset H$ the indetermination set of $f$, i.e., the set of points in the neighborhood of which $f$ is not holomorphic. We denote $X_{f}:=\overline{f\left(H \backslash I_{f}\right)} \subset H$. This is an analytic set (see for example [14] for details).

Let $\omega$ be a Kählerian form of mass 1 on $\mathbb{P}^{n}$. We define the dynamical degrees $\lambda_{1}(f), \ldots, \lambda_{n}(f) \geq 1$ of $f$ by

$$
\lambda_{k}(f):=\liminf _{m \rightarrow+\infty}\left(\int_{\mathbb{C}^{n}}\left(f^{m *} \omega^{k}\right) \wedge \omega^{n-k}\right)^{\frac{1}{m}} .
$$

Note that they are independent of the choice of the Kähler form $\omega$. When there is no risk of ambiguity, we will simply denote them by $\lambda_{k}$, and put $\lambda:=\lambda_{1}$.

The pull-back operation of a current induces a linear action $f^{*}$ on the cohomology spaces, defined by $f^{*}\{S\}:=\left\{f^{*} S\right\}$. A polynomial application $f: \mathbb{C}^{n} \rightarrow \mathbb{C}^{n}$ extended to $\mathbb{P}^{n}$ is called algebraically stable if the linear action induced by $f^{*}$ on the cohomology spaces $H^{k, k}\left(\mathbb{P}^{n}, \mathbb{R}\right)$ (of dimension 1) commutes with the composition operation, i.e., when $\left(f^{n}\right)^{*}=\left(f^{*}\right)^{n}$ for all $n \in \mathbb{N}$. When $f$ is algebraically stable, the spectral radius of the linear action induced in cohomology $f^{*}: H^{k, k}\left(\mathbb{P}^{n}, \mathbb{R}\right) \rightarrow H^{k, k}\left(\mathbb{P}^{n}, \mathbb{R}\right)$ equals the $k$-th dynamical degree $\lambda_{k}$. By duality, it also equals the spectral radius $\lambda_{n-k}^{\prime}$ of the action induced by $f_{*}$ on $H^{n-k, n-k}\left(\mathbb{P}^{n}, \mathbb{R}\right)$. In order for $f$ to be algebraically stable, it suffices to have $I_{f} \cap X_{f}=\emptyset$.

We will be interested in polynomial applications verifying $\lambda_{1}>\lambda_{2}$, whose properties will be seen to generalize those of the Hénon's applications of $\mathbb{C}^{2}$. The concavity inequalities fulfilled by the $\lambda_{i}$ (Theorem 2.4.a in [14]) imply that $\lambda_{1}$ is a dynamical degree strictly dominant $\left(\lambda_{1}>\max _{j \neq 1} \lambda_{j}\right)$.

Given an algebraically stable polynomial endomorphism $f$ of $\mathbb{P}^{n}$ of dynamical degree $\lambda_{1}$ strictly dominant, and $\omega$ a Kählerian form of mass 1 , the sequence $\frac{1}{\lambda_{1}{ }^{m}} f^{m *} \omega$ converges, in the weak sense of currents, toward a closed positive $(1,1)$-current $T^{+}$(see, e.g., Theorem 1.1 in [2] for a general statement). The current $T^{+}$is invariant by $\frac{1}{\lambda_{1}} f^{*}$ and does not depend on the choice of $\omega$. We denote by $J$ its support.

If moreover $f$ is an automorphism, we can define a canonical positive $(n-1, n-1)$ current $\frac{1}{\lambda_{1}} f_{*}$-invariant $T^{-}:=\lim _{m \rightarrow+\infty} \frac{1}{\lambda_{1}{ }^{m}} f^{m}{ }_{*} \omega^{n-1}$. The measures $\left\|T^{+}\right\|$and $\left\|T^{-}\right\|$do not charge pluripolar sets.

More generally, even if $f$ is not an automorphism, the current $T^{-}$is still well defined if $f$ satisfies $\lim _{z \in \mathcal{C}_{f},\|z\| \rightarrow+\infty} f(z) \in X_{f}$, where $\mathcal{C}_{f}$ is the critical set of $f$ (see section 2.2 in [16], see also [15]).

The canonical invariant measure $T^{+} \wedge T^{-}$is then well defined. It does not charge pluripolar sets. We will denote by $J^{-}$the support of such current $T^{-}$.

If $f$ is a polynomial endomorphism of $\mathbb{P}^{n}$ which is algebraically stable and verifies $\lambda_{1}>$ $\lambda_{2}$, then there exists a plurisubharmonic function $G$ defined on $\mathbb{C}^{n} \subset \mathbb{P}^{n}$, called dynamical Green's function of $f$, such that $T^{+}=d d^{c} G$. It can be written as the limit in $L_{\text {loc }}^{1}$ of the sequence of term $\frac{1}{\lambda_{1} m} \log \left(1+\left\|f^{m}\right\|\right)$. The function $G$ is continuous and pluriharmonic outside the support $J$ of $T^{+}$.

The current $T^{+}$intersects the divisor at infinity exactly on the indetermination set of $f$, i.e., on the points where $f$ cannot be holomorphically extended at infinity. This indetermination 
set is an analytical subset of $\mathbb{P}^{n}$ of (complex) dimension at most $n-2$. (See Theorem 4.17 in [14] for more details.)

The invariance property $G \circ f=\lambda_{1} G$ implies that $f$ sends the sublevel set $\{G<\epsilon\}$ onto the sublevel set $\left\{G<\lambda_{1} \epsilon\right\}$. In other words, the set $K^{+}:=\{G=0\}$, which we call filled Julia set, is included in an open set dilated by $f$. Therefore, every $y \notin K^{+}$verifies $\lim _{m \rightarrow+\infty}\left\|f^{m}(y)\right\|=+\infty$. We thus define:

Definition 3 A polynomial automorphism $f: \mathbb{C}^{n} \rightarrow \mathbb{C}^{n}, n \geq 2$, algebraically stable and such that $\lambda_{1}>\lambda_{2}$, will be called quasi-Hénon if $X_{f} \cap I_{f}=\emptyset$ and the set $I_{f}$ is $f^{-1}$-attracting.

Example 1 The Hénon's applications $f(x, y)=\left(x^{2}+a y, x\right), a \in \mathbb{C}^{*}$ are quasi-Hénon automorphisms of $\mathbb{C}^{2}$ if $|a|<1$.

In the following subsections, $f: \mathbb{C}^{n} \rightarrow \mathbb{C}^{n}$ will be supposed to be a quasi-Hénon map. We then have $\lambda_{n}=1$ and $f_{*}=\left(f^{-1}\right)^{*}$ on $\mathbb{C}^{n}$.

\subsection{Equidistribution result for currents}

The following result, which generalizes a theorem of [3] (see also Theorem 7.17 in [12]), will allow us to study locally the current $d d^{c} G$ in dimension $n>1$.

Lemma 7 Let $f: \mathbb{C}^{n} \rightarrow \mathbb{C}^{n}$ be a quasi-Hénon map. Let $S$ be a closed positive $(1,1)$-current on $\mathbb{P}^{n}$, with mass 1 , without Lelong numbers, and let $\xi \geq 0$ be a $C^{\infty}$ function of compact support in $\mathbb{C}^{n} \subset \mathbb{P}^{n}$. Then, denoting $c:=\int_{\mathbb{P}^{n}} \xi S \wedge T^{-} \geq 0$, we have

$$
\lim _{m \rightarrow+\infty} \frac{1}{\lambda_{1}{ }^{m}} f^{m *}(\xi S)=c \cdot T^{+}
$$

On the other hand, denoting $c^{\prime}:=\int_{\mathbb{P}^{n}} \xi S \wedge T^{+} \geq 0$, we have

$$
\lim _{m \rightarrow+\infty} \frac{1}{\lambda_{1}{ }^{m}} f_{*}^{m}\left(\xi T^{-}\right)=\lim _{m \rightarrow+\infty} \frac{1}{\lambda_{1}{ }^{m}} f_{*}^{m}\left(\xi \omega^{n-l}\right)=c^{\prime} \cdot T^{-} .
$$

Proof We will give the proof in the case $n>2$, the case $n=2$ being only a simplified version. We may suppose, without loss of generality, that $0<\xi \leq 1$. Let $\omega$ be the Fubiny-Study $(1,1)$-form. Let $\phi$ be a closed $(0,1)$-form.

We put $S_{m}:=\frac{1}{\lambda_{1}{ }^{m}} f^{m *}(\xi S)$. Let us first show that the sequence of currents $\left(S_{m}\right)_{m}$ is bounded. It follows, using the fact that the currents $\frac{1}{\lambda_{1}{ }^{m}} f_{*}^{m} \omega^{n-1}$ are cohomologous, that $\forall m \in \mathbb{N}$,

$$
\int_{\mathbb{P}^{n}} S_{m} \wedge \omega=\int_{\mathbb{P}^{n}} \xi S \wedge \frac{1}{\lambda_{1}{ }^{m}} f_{*}^{m} \omega^{n-1} \leq \int_{\mathbb{P}^{n}} S \wedge \frac{1}{\lambda_{1}{ }^{m}} f_{*}^{m} \omega^{n-1}=\int_{\mathbb{P}^{n}} S \wedge \omega^{n-1}<+\infty .
$$

The mass of the currents $S_{m}$ is thus uniformly bounded. 
Let us now show that an accumulation point $S_{\infty}$ of the sequence $\left(S_{m}\right)_{m}$ is closed. The Cauchy-Schwarz inequality gives:

$$
\begin{aligned}
\lim _{m \rightarrow+\infty}\left|\int_{\mathbb{P}^{n}} \partial S_{m} \wedge \phi \wedge \omega^{n-2}\right|=\lim _{m \rightarrow+\infty} \frac{1}{\lambda_{1} m}\left|\int_{\mathbb{C}^{n}} \partial \xi \wedge S \wedge f_{*}^{m}\left(\omega^{n-2}\right) \wedge f_{*}^{m} \phi\right| \\
\leq \lim _{m \rightarrow+\infty} \frac{1}{\lambda_{1}^{m}}\left|\int_{\mathbb{C}^{n}} f_{*}^{m}\left(\omega^{n-2}\right) \wedge S \wedge \partial \xi \wedge i \bar{\partial} \xi\right|^{1 / 2} \cdot\left|\int_{\mathbb{C}^{n}} f_{*}^{m}\left(\omega^{n-2} \wedge \phi \wedge i \bar{\phi}\right) \wedge S\right|^{1 / 2} \\
=\lim _{m \rightarrow+\infty}\left(\frac{\lambda_{n-2}^{\prime}}{\lambda_{1}}\right)^{m / 2}\left|\int_{\mathbb{C}^{n}}\left(\frac{1}{\lambda_{n-2}^{\prime}}\right)^{m} f_{*}^{m}\left(\omega^{n-2}\right) \wedge S \wedge \partial \xi \wedge i \bar{\partial} \xi\right|^{1 / 2} \\
\left.\cdot\left|\int_{\mathbb{C}^{n}}\left(\frac{1}{\lambda_{1}}\right)^{m} f_{*}^{m}\left(\omega^{n-2} \wedge \phi \wedge i \bar{\phi}\right) \wedge S\right|^{1 / 2}\right|_{m \rightarrow+\infty}\left(\frac{\lambda_{2}}{\lambda_{1}}\right)^{m / 2}\left|\int_{\mathbb{C}^{n}}\left(\frac{1}{\lambda_{2}}\right)^{m} f_{*}^{m}\left(\omega^{n-2}\right) \wedge S \wedge \partial \xi \wedge i \bar{\partial} \xi\right|^{1 / 2} \\
\quad \cdot\left|\int_{\mathbb{C}^{n}} \omega^{n-2} \wedge \phi \wedge i \bar{\phi} \wedge\left(\frac{1}{\lambda_{1}}\right)^{m} f^{m *} S\right|^{1 / 2} \\
=\lim _{m \rightarrow+\infty} O\left(\left(\frac{\lambda_{2}}{\lambda_{1}}\right)^{m / 2}\right)=0 .
\end{aligned}
$$

We will further show that $S_{\infty}=c \cdot T^{+}$. By virtue of Theorem 1.1 in [2], we have $\frac{1}{\lambda_{1}{ }^{m}} f^{m *} S \longrightarrow T^{+}$in the sense of currents. Since $S_{m} \leq \frac{1}{\lambda_{1}{ }^{m}} f^{m *} S$ for all $m$, due to the positivity of the operator $f^{*}$, we have $S_{\infty} \leq T^{+}$. The extremality of $T^{+}$(Theorem 2.1 in [16]) leads to $S_{\infty}=c \cdot T^{+}$, with $c:=\int_{\mathbb{P} n} \xi S \wedge T^{-} \geq 0$. (Note that for $n=2$, the assumption that $S$ does not have Lelong numbers can be removed thanks to Theorem A in [23].)

In order to show that $\lim _{m \rightarrow+\infty} \frac{1}{\lambda_{1}{ }^{m}} f_{*}{ }^{m}(\xi S)=c \cdot T^{-}$when $S=\omega$ or $T^{-}$, the proof proceeds similarly, with the following precision. If $S_{\infty}$ is an accumulation point of the sequence of term $\frac{1}{\lambda_{1}{ }^{m}} f_{*}{ }^{m}(\xi S)$, then $S_{\infty}=c^{\prime} \cdot T^{-}$follows from Theorem 2.6 in [16].

Let us give some consequences. Recall that we denote $K^{+}:=\{G=0\}$.

Proposition 5 Let $f: \mathbb{C}^{n} \rightarrow \mathbb{C}^{n}, n \geq 1$ be a quasi-Hénon map. Then, $\partial K^{+}$is the support of $T^{+}$.

Proof Recall that we denote $J:=\operatorname{supp}\left(T^{+}\right)=\operatorname{supp}\left(d d^{c} G\right)$. Note that the sets $K^{+}, \partial K^{+}$ and $J$ are invariant by $f$ and by $f^{-1}$.

There exists a $C^{\infty}$ function $\xi \geq 0$ with compact support in $\stackrel{\circ}{K}^{+}$whose interior intersects $J^{-}$. Using Lemma 7, if $\omega$ is the Fubiny-Study $(1,1)$-form, there exists $c>0$ such that:

$$
\lim _{m \rightarrow+\infty} \frac{1}{\lambda_{1}{ }^{m}} f^{m *}(\xi \omega)=c \cdot T^{+} .
$$

It follows that $J \subset K^{+}$, and more precisely that $J \subset \partial K^{+}$.

Conversely, suppose by contradiction that there exists $x \in \partial K^{+} \backslash J$. The function $G$ is harmonic in a small neighborhood $W$ of $x$, where it has a minimum $G(x)=0$, hence it vanishes in $W$, which is impossible. Thus, $J=\partial K^{+}$.

Lemma 8 The filled Julia set $K^{+}$of a quasi-Hénon map $f: \mathbb{C}^{n} \rightarrow \mathbb{C}^{n}, n \geq 2$, if it is of non-empty interior, satisfies $\overline{K^{+}}=K^{+}$. 
Proof Let $\xi \omega$ be a $(1,1)$-form of support in $\stackrel{\circ}{K}^{+}$, where $\omega$ is the Fubini-Study form and $\xi \geq 0$ is a $C^{\infty}$ function of compact support in $K^{+}$intersecting $J^{-}$. Note that $K^{+}$is invariant by $f$ and by $f^{-1}$, thus $\stackrel{\circ}{K}^{+}$is also. Note that, by Proposition $5, J=\partial K^{+}$. The support of $f^{m *}(\xi \omega)$ being in $\stackrel{\circ}{K}^{+}$for all $m$, by Lemma 7, every open subset of $\mathbb{C}^{n}$ intersecting $J=\partial K^{+}$also intersects $\stackrel{\circ}{K}^{+}$, thus $\overline{K^{+}}=K^{+}$.

\subsection{Equidistribution result for measures}

We give conditions under which the means of pull-backs $\mu_{m}:=\frac{1}{m} \sum_{i=0}^{m} f^{i *} \mu$ converge to the invariant measure $T^{+} \wedge T^{-}$. Recall that we denote by $J$ and, respectively, $J^{-}$the supports of the currents $T^{+}$and $T^{-}$.

Lemma 9 Let $f: \mathbb{C}^{n} \rightarrow \mathbb{C}^{n}$ be a quasi-Hénon map. Let $U$ be an open set intersecting $J \cap J^{-}$. Then, $\bigcup_{m \geq 0} f^{m}\left(\left.U\right|_{J^{-}}\right)$, and even $\bigcap_{i \geq 0} \bigcup_{m \geq i} f^{m}\left(\left.U\right|_{J^{-}}\right)$, are dense in $J^{-}$for the induced topology.

More precisely, for every open set $V$ intersecting $J^{-}$, there exists $\epsilon>0$ such that

$$
\left\|T^{-}\right\|\left(f^{m}\left(\left.U\right|_{J^{-}}\right) \cap V\right)>\epsilon
$$

for $m$ sufficiently large.

Proof Let $\xi$ be a positive $\mathcal{C}^{\infty}$ function with compact support $Z$, such that $U:=\dot{Z}$ intersects $\operatorname{supp}\left(T^{+} \wedge T^{-}\right) \subset J \cap J^{-}$. By Lemma 7 , the sequence of currents $\left(\frac{1}{\lambda_{1}{ }^{m}} f^{m} *\left(\xi T^{-}\right)\right)_{m}$ converges to $c \cdot T^{-}$, with $c=\int \xi T^{-} \wedge T^{+}>0$, when $m \rightarrow+\infty$.

But the support of $\frac{1}{\lambda_{1}{ }^{m}} f^{m} *\left(\xi T^{-}\right)$is $f^{m}(Z) \cap J^{-}$, hence for every open set $V$ intersecting $J^{-}, \exists m_{0} \in \mathbb{N}$ such that $f^{m}\left(\left.U\right|_{J^{-}}\right) \cap V \neq \emptyset$ when $m \geq m_{0}$. In other words, $f^{m}\left(\left.U\right|_{J^{-}}\right) \cap$ $\left.V\right|_{J^{-}} \neq \emptyset$ when $m \geq m_{0}$. Therefore, $\bigcup_{m \geq 0} f^{m}\left(\left.U\right|_{J^{-}}\right)$is dense in $J^{-}$for the induced topology, and even $\bigcap_{i \geq 0} \bigcup_{m \geq i} f^{m}\left(\left.U\right|_{J^{-}}\right)$.

More precisely, it follows from the convergence of $\left(\frac{1}{\lambda_{1}{ }^{m}} f^{m} *\left(\xi T^{-}\right)\right)_{m}$ to $c \cdot T^{-}$when $m \rightarrow+\infty$ that there exists $\epsilon>0$ such that $\left\|T^{-}\right\|\left(f^{m}\left(\left.U\right|_{J^{-}}\right) \cap V\right)>\epsilon$ when $m \geq m_{0}$. Indeed, let us suppose that $\max _{x \in \mathbb{C}^{n}} \xi(x)=1$, and consider a test function $\rho$ positive on $V$, such that $\max _{x \in \mathbb{C}^{n}} \rho(x)=1$. Then,

$$
\begin{aligned}
& \left\|T^{-}\right\|\left(f^{m}\left(\left.U\right|_{J^{-}}\right) \cap V\right)=\frac{1}{\lambda_{1}{ }^{m}} \int_{f^{m}(U) \cap V}\left(f^{m}{ }_{*} T^{-}\right) \wedge \omega \geq \frac{1}{\lambda_{1}{ }^{m}} \int_{\mathbb{C}^{n}}\left(f^{m}{ }_{*} \xi T^{-}\right) \wedge \rho \omega \\
& \underset{m \rightarrow+\infty}{\longrightarrow} c \int_{\mathbb{C}^{n}} T^{-} \wedge \rho \omega>0 .
\end{aligned}
$$

We can now give the following convergence result (see for example [30] or [19] for the definition of an ergodic measure). The fact that the support of $T^{+} \wedge T^{-}$is $J \cap J^{-}$is proved in dimension 2 in [10] in a little different context.

Proposition 6 Let $f: \mathbb{C}^{n} \rightarrow \mathbb{C}^{n}$ be a quasi-Hénon map. If $\mu$ is a probability measure with compact support on $J^{-}$that does not charge pluripolar sets, then the sequence of measures $\left(\mu_{m}:=\frac{1}{m} \sum_{i=0}^{m} f^{i *} \mu\right)_{m}$ converges weakly to the invariant probability measure $T^{+} \wedge T^{-}$. Moreover, the measure $T^{+} \wedge T^{-}$is ergodic, and its support is the compact set $J \cap J^{-}$. 
Proof Let us denote by $\mu_{f}$ an accumulation point of the sequence of probability measures $\left(\frac{1}{m} \sum_{i=0}^{m} f^{i *} \mu\right)_{m}$. By construction, we have $f^{*} \mu_{f}=\mu_{f}$, with $\operatorname{supp}\left(\mu_{f}\right) \subset J \cap J^{-}$. It remains to show the inclusion in the opposite direction.

(1) Let us show that the support of $T^{+} \wedge T^{-}$is exactly $J \cap J^{-}$. Let $U \subset \mathbb{C}^{n}$ be an open set intersecting $J \cap J^{-}$. Denote by $\xi$ a positive test function such that $\xi \equiv 1$ on $U$. By Lemma 7, we have

$$
\frac{1}{\lambda_{1}^{m}} f_{*}^{m}\left(\xi T^{-}\right) \rightarrow c \cdot T^{-}
$$

for a constant $c \geq 0$. We will show that $c>0$. Since $c=\int_{\mathbb{C}^{n}} \xi d\left(T^{+} \wedge T^{-}\right)$, this will imply that the support of $T^{+} \wedge T^{-}$is included in $J \cap J^{-}$.

Note that $\forall m \in \mathbb{N}$, we have

$$
\frac{1}{\lambda_{1}{ }^{m}} f_{*}^{m}\left(\xi T^{-}\right) \geq\left.\frac{1}{\lambda_{1}{ }^{m}} f^{m}{ }_{*} T^{-}\right|_{U}=\left.T^{-}\right|_{f^{m}(U)} .
$$

But by Lemma 9, for every open set $V$ intersecting $J^{-}, \exists \epsilon>0$, such that for $m$ sufficiently large, we have

$$
\left\|T^{-}\right\|\left(f^{m}\left(\left.U\right|_{J^{-}}\right) \cap V\right)>\epsilon .
$$

Thus, $c>0$. Finally $\operatorname{supp}\left(T^{+} \wedge T^{-}\right) \subset J \cap J^{-}$. The inverse inclusion is trivial.

(2) Let $\rho$ be a test function whose support $U$ intersects $J^{+} \cap J^{-}$. We can decompose $\rho$ into the difference of two positive continuous plurisubharmonic functions $\rho=u-v$. (For example, we can choose $u=\rho+A \log \left(1+\|z\|^{2}\right)$ and $v=A \log \left(1+\|z\|^{2}\right)$ for $A$ sufficiently large.) Consider the sequence of functions $\left(\phi_{m}\right)_{m}:=\left(\left.\frac{1}{m} \sum_{i=1}^{m} f^{i}{ }_{*} u\right|_{J^{-}}\right)_{m}$ defined on $\mathcal{S}$.

Note that this sequence is bounded on every compact of $J^{-}$: indeed, let $U$ be an open set containing $J \cap J^{-}$, and denote $H$ the compact set $J^{-} \cap\{G=0\}$. Then, $\left.U\right|_{J^{-}} \subset \subset$ $\left.f(U)\right|_{J^{-}} \cup H$. Consequently, we have $\forall m \in \mathbb{N}$, $\sup _{\left.U\right|_{J^{-}}} \phi_{m} \leq \sup _{\left.U\right|_{J^{-}} \cup H} u$.

Denote $\phi_{\infty}:=\limsup _{m \rightarrow+\infty} \phi_{m}$. Then, by Lemma $2, \phi_{\infty}^{*}$ is a $T$-psh function. Since $\phi_{\infty}$ satisfies by construction $f_{*} \phi_{\infty}=\phi_{\infty}$, we have by Lemma 2 :

$$
f_{*} \phi_{\infty}^{*}=\phi_{\infty}^{*} .
$$

(3) Let us show that $\phi_{\infty}^{*}$ is constant on $J^{-}$. Let $c:=\max _{J \cap J^{-}} \phi_{\infty}^{*}$ and $\epsilon>0$. Then, $J \cap J^{-}$is included in the open set $U:=\left\{\phi_{\infty}^{*}<c+\epsilon\right\}$. Then, (11) implies that $\forall m \in \mathbb{N}$,

$$
f^{m}(U)=\left\{f^{m}{ }_{*} \phi_{\infty}^{*}<c+\epsilon\right\}=U .
$$

We have $\phi_{\infty}^{*} \leq c+\epsilon$ on $U=\bigcup_{m>0} f^{m}(U)$, and this open set being dense in $J^{-}$for the induced topology by Lemma 9, Theorem 3 and Corollary 3 imply $\phi_{\infty}^{*} \leq c+\epsilon$ on $J^{-}$. The constant $\epsilon>0$ being arbitrary, it follows that $\phi_{\infty}^{*} \leq c$ on $J^{-}$. Instead of invoking Proposition 3, let us pursue with some dynamical arguments.

Let $d:=\inf _{J \cap J^{-}} \phi_{\infty}^{*}$ and $\epsilon>0$. Let $V:=\left\{\phi_{\infty}^{*}<d-\epsilon\right\}$. Then, $J \cap J^{-} \cap \bar{V}=\emptyset$. As previously we have $V=f(V)$; since for any bounded set $W$ containing $J \cap J^{-}$there exists $m_{0} \in \mathbb{N}$ such that $f^{m}(V) \cap W \neq \emptyset$ for $m \geq m_{0}$, we obtain $V=\emptyset$. Therefore, $\phi_{\infty}^{*} \geq d$ on $J^{-}$.

If $\left.\phi_{\infty}^{*}\right|_{J \cap J^{-}}$is not constant on $J \cap J^{-}$, there exists $\epsilon>0$ such that the open set $W:=$ $\left\{\phi_{\infty}^{*}<c-\epsilon\right\}$ is non-empty and intersects $J \cap J^{-}$. Moreover, we have again $f(W)=W$. By Lemma 9, $W=\bigcup_{m \geq 0} f^{m}(W)$ is an open set dense in $J^{-}$. By Theorem 3 and Corollary 3 , 
$\phi_{\infty}^{*}<c-\epsilon$ in $J \cap J^{-}$, which contradicts the definition of $c:=\max _{J \cap J^{-}} \phi_{\infty}^{*}$. Therefore, $\phi_{\infty}^{*}=c$ on $J \cap J^{-}$. Thus, $c=d$, hence $\phi_{\infty}^{*}=c$ on $J^{-}$.

(4) Conclusion. The sequence $\left(\phi_{m}\right)_{m}$ converges in $L^{1}\left(T^{+} \wedge T^{-}\right)$by Birkhoff's ergodic theorem. The set $\left\{\phi_{\infty}<\phi_{\infty}^{*}\right\}$ is pluripolar by Lemma 6 . Since the measure $T^{+} \wedge T^{-}$does not charge pluripolar sets, we deduce that the sequence $\left(\phi_{m}\right)_{m}$ converges $T^{+} \wedge T^{-}$-a.e. toward $c=\limsup _{m \rightarrow+\infty} \phi_{m}$. Proposition 3 implies that this sequence converges $\left\|T^{-}\right\|$-a.e. toward $c$ on $J^{-}$. Let us denote by $\left(\mu_{m_{i}}\right)_{i}$ a sequence of measures, extracted from $\left(\mu_{m}\right)_{m}$, which converges to $\mu_{f}$. Since the measure $\mu$ does not charge, by hypothesis, the pluripolar sets, thanks to the dominated convergence theorem, there exists a constant $C \in \mathbb{R}$ such that

$$
\left\langle\mu_{f}, \rho\right\rangle=\lim _{i \rightarrow+\infty}\left\langle\mu, \frac{1}{m_{i}} \sum_{j=1}^{m_{i}} f^{j}{ }_{*} u\right\rangle-\lim _{i \rightarrow+\infty}\left\langle\mu, \frac{1}{m_{i}} \sum_{j=1}^{m_{i}} f^{j}{ }_{*} v\right\rangle=C .
$$

On the other hand, since the measure $T^{+} \wedge T^{-}$does not charge pluripolar sets (see, e.g., [14]), we have

$$
\begin{aligned}
\left\langle T^{+} \wedge T^{-}, \rho\right\rangle= & \lim _{i \rightarrow+\infty}\left\langle T^{+} \wedge T^{-}, \frac{1}{m_{i}} \sum_{j=1}^{m_{i}} f^{j}{ }_{*} u\right\rangle \\
& -\lim _{i \rightarrow+\infty}\left\langle T^{+} \wedge T^{-}, \frac{1}{m_{i}} \sum_{j=1}^{m_{i}} f^{j}{ }_{*} v\right\rangle=C .
\end{aligned}
$$

Thus, $T^{+} \wedge T^{-}=\mu_{f}$. The ergodicity of this invariant measure follows directly from Theorem 4.4.1 in [19].

Remark 6 Let us emphasize that, in the proof of Proposition 6, we have obtained that if $\rho$ is a test function, the sequence of functions $\left(\frac{1}{m} \sum_{i=1}^{m} f^{i}{ }_{*} \rho\right)_{m}$ converges toward a constant $C$ outside a pluripolar set, and therefore converges in $L_{\text {loc }}^{1}\left(T^{+} \wedge T^{-}\right)$toward $C$ thanks to the dominated convergence theorem. Moreover, $C>0$ if and only if the support of $\rho>0$ intersects $J \cap J^{-}$.

We thus deduce an alternative proof of the point 1) of the proof of Proposition 6 above. Let $\xi>0$ be a test function with support intersecting $J \cap J^{-}$. Then, $\forall m \in \mathbb{N}$,

$$
\int_{\mathbb{C}^{n}} \xi d\left(T^{+} \wedge T^{-}\right)=\int_{\mathbb{C}^{n}} \frac{1}{m} \sum_{i=1}^{m} f_{*}^{i} \xi d\left(T^{+} \wedge T^{-}\right) .
$$

By taking $m \rightarrow+\infty$, since $T^{+} \wedge T^{-}$does not charge pluripolar sets, the dominated convergence theorem implies that $\int_{\mathbb{C}^{n}} \xi d\left(T^{+} \wedge T^{-}\right)>0$, which concludes.

The following remark generalizes Theorem 6.6 in [13].

Remark 7 We deduce from Remark 6 that for every open set $U$ intersecting $J \cap J^{-}$, the set $J^{-} \backslash \bigcup_{m \geq 0} f^{m}\left(\left.U\right|_{J^{-}}\right)$is pluripolar.

Proof Indeed, let $\rho$ be a strictly positive test function of support included in $U$, and whose interior intersects $J \cap J^{-}$. Then,

$$
\phi_{\infty}:=\limsup _{m \rightarrow+\infty} \frac{1}{m} \sum_{i=1}^{m} f_{*}^{i} \rho \leq \limsup _{m \rightarrow+\infty} \frac{1}{m} \sum_{i=1}^{m} f^{i}{ }_{*} \mathbf{1}_{U} .
$$


On the other hand, Remark 6 shows that $\phi_{\infty}$ is equal, outside a pluripolar set, to a constant $C>0$. Thus,

$$
J^{-} \backslash \bigcup_{m \geq 0} f^{m}\left(\left.U\right|_{J^{-}}\right) \subset\left\{\limsup _{m \rightarrow+\infty} \frac{1}{m} \sum_{i=1}^{m} f^{i}{ }_{*} \mathbf{1}_{U}=0\right\} \subset\left\{\phi_{\infty}=0\right\} \subset\left\{\phi_{\infty}<C\right\} .
$$

Thus, all these sets are pluripolar, which proves the statement.

Acknowledgements We thank Prof. E. Bedford and C. Kiselman for their attentive reading.

\section{References}

1. Alexander, H.: Projective capacity. Ann. Math. Stud. 100, 3-27 (1981)

2. Bayraktar, T.: Equidistribution towards the Green current in big cohomology classes. Int. J. Math. 24, 1350080 (2013)

3. Bedford, E., Smillie, J.: Polynomial diffeomorphisms of $\mathbb{C}^{2}$. II. J. Am. Math. Soc. 4, 657-679 (1991)

4. Demailly, J.P.: Monge-Ampere operators, Lelong numbers, and intersection theory. In: Ancona, V., Silva, A. (eds.) Complex Analysis and Geometry, pp. 115-191. Plenum Press, New York (1993)

5. Dinh, T.C., Dujardin, R., Sibony, N.: On the dynamics near infinity of some polynomial mappings in $\mathbb{C}^{2}$. Math. Ann. 333, 703-739 (2005)

6. Dinh, T.C., Lawrence, M.G.: Polynomial hulls and positive currents. Ann. Fac. Sci. Toulouse Math. series 6(12), 317-334 (2003)

7. Dinh, T.C., Sibony, N.: Dynamique des applications polynomiales semi-régulières. Ark. Mat. 42, 61-85 (2004)

8. Duval, J., Sibony, N.: Polynomial convexity, rational convexity, and currents. Duke Math. J. 79, 487-513 (1995)

9. Edwards, D.A.: Choquet boundary theory for certain spaces of lower semicontinuous functions. In: Birtel (Ed.) Function Algebras. Scott Foresman and Cie, pp. 300-309 (1966)

10. Fornaess, J.E.: The Julia set of Hénon maps. Math. Ann. 334, 457-464 (2006)

11. Favre, C., Guedj, V.: Dynamique des applications rationnelles des espaces multiprojectifs. Indiana Univ. Math. J. 50, 881-934 (2001)

12. Fornaess, E., Sibony, N.: Complex dynamics in higher dimensions. In: Complex Potential Theory, NATO ASI series C, 439, pp. 131-18. Gauthier ed. 6 (1994)

13. Fornaess, E., Sibony, N.: Complex dynamics in Higher dimensions II. In: Modern Methods in Complex Analysis, pp. 135-182. Annals of Mathematics Studies 137,(1995)

14. Guedj, V.: Propriétés ergodiques des applications rationnelles. In: Quelques aspects des systèmes dynamiques polynomiaux. Panor. Synthèses 30, 97-202. Soc. Math. France, Paris (2010)

15. Guedj, V., Sibony, N.: Dynamics of polynomials automorphisms of $\mathbb{C}^{k}$. Ark. Mat. 40, 207-243 (2002)

16. Guedj, V.: Courants extrémaux et dynamique complexe. Ann. Sci. École Norm. Sup. 38, 407-426 (2005)

17. Gamelin, T.W., Rossi, H.: Jensen measures and algebras of analytic functions. In: Birtel, F.T. (Ed.) Function Algebras, pp. 15-35. Scott Foresman and Cie (1966)

18. Klimek, M.: Pluripotential Theory. Clarendon Press, Oxford (1991)

19. Lasota, A., Mackey, M.C.: Probabilistic Properties of Deterministic Systems. Cambridge University Press, Cambridge (1985)

20. Poletsky, E.A.: Plurisubharmonic functions as solutions of variational problems. In: Several Complex Variables and Complex Geometry (Santa Cruz, CA, 1989), Part. 1, pp. 163-171. American Mathematical Society, Providence (1991)

21. Poletsky, E.A.: Holomorphic currents. Indiana Univ. Math. J. 42, 85-144 (1993)

22. Protin, F.: Dynamique d'endomorphismes polynomiaux de $\mathbb{C}^{k}, \mathrm{PhD}$ thesis, Université Toulouse III (2010)

23. Protin, F.: Equidistribution vers le courant de Green. Ann. Polon. Math. 115, 201-218 (2015)

24. Protin, F.: $Ł S$ condition for filled Julia sets in $\mathbb{C}$. Annali di Matematica (2018). https://doi.org/10.1007/ s10231-018-0752-X

25. Ransford, T.J.: Jensen measures. In: Arakelian, N., Gauthier, P.M. (eds.) Approximation, Complex Analysis and Potential Theory, pp. 221-237. Kluwer, Dordrecht (2001)

26. Rosay, J.P.: Poletsky theory of disks on holomorphic manifolds. Indiana Univ. Math. J. 52, 157-169 (2003)

27. Sibony, N.: Pfaff systems, currents and hulls. Math. Z. 285, 1107-1123 (2017) 
28. Stout, E.L.: Polynomial Convexity, Progress in Mathematics, 261. Birkaüser, Basel (2007)

29. Stout, E.L.: The Theory of Uniform Algebras. Bogden and Quigley (1971)

30. Walters, P.: An Introduction to Ergodic Theory. Springer, Berlin (1981)

Publisher's Note Springer Nature remains neutral with regard to jurisdictional claims in published maps and institutional affiliations. 